\title{
ADDITIVITY OF CHAIN-LADDER PROJECTIONS
}

\author{
BY BJÖRN AJNE \\ Skandia, Stockholm
}

\begin{abstract}
In this paper some results are given on the addivity of chain-ladder projections. Given two claims development triangles, when do their chain-ladder projections add up to the projections of the combined triangle, that is the triangle being the element-wise sum of the two given triangles?

Necessary and sufficient conditions for equality are given. These are of a fairly simply form and are directly connected to the ordinary chain-ladder calculations. In addition, sufficient conditions of the same form are given for inequality between the combined projection vector and the sum of the two original projections vectors.
\end{abstract}

\section{KEYWORDS}

Chain-ladder projections.

\section{INTRODUCTION}

Consider two claims development triangles $C$ and $D$. $C$ consists of positive elements $C(i, j)$, where $i$ denotes the accident years and runs from 0 to $n$. The index $j$ denotes the development year. For each $i$ it runs from 0 to $n-i$. Thus $n$ denotes the calendar year at the end of which the triangle $C$ is observed, the oldest accident year observed being year number zero. For $D$ the same things hold true with $C(i, j)$ exchanged for $D(i, j)$.

The triangles $C$ and $D$ are thought of as corresponding to two different subportfolios. The elements $C(i, j)$ and $D(i, j)$ are thought of as accumulated claims data for accident year $i$ at the end of development year $j$, be it claims numbers or claims payments or payments plus known reserves. Below they are referred to as amounts.

If now we fill out the triangles into full squares using the ordinary chain-ladder method, $C(i, n)$ and $D(i, n)$ will for each accident year $i$ be the projected final accumulated amounts for that year. $C(0, n)$ and $D(0, n)$ are already there, being the final amounts for the base year. Adding $C(i, n)$ and $D(i, n)$ for all $i$, we will get the projected final accumulated amounts for the combined portfolio. 
This, however, we can also get in another way. We can add the two triangles $C$ and $D$ to get a third triangle $E$ with elements $E(i, j)$, being sums of the corresponding $C(i, j)$ and $D(i, j)$. Then we do the chain-ladder on $E$ to obtain projected final accumulated amounts $E(i, n)$ for the combined portfolio.

The purpose of this paper is to study under what circumstances the two methods will give the same result. This is done in Section 3. When these circumstances are not present sufficient conditions will be given for one method to be more prudent than the other one. This is done in Section 4.

The paper is an improved version of a paper presented to the 23rd ASTIN Colloquium (AJNE, 1991) with simpler proofs and somewhat more far-reaching results.

The practical application is rather the opposite way round to that described above. We are given the total portfolio. When should we contemplate dividing it up into subportfolios in order to get more prudent estimates of its final amounts?

In the appendix an illustration is given in the form of four pairs of simple development triangles $(C, D)$.

Among other things, the question of additivity of claims reserving methods is treated in an lecture given by Hans Bühlmann at the 24th ASTIN Colloquium in Cambridge (BüHLMAnN, 1993).

\section{SOME CHAIN-LADDER FORMULAS}

Let us recall some chain-ladder calculations. We do it for the triangle $C$, the corresponding being valid for $D$ and $E$.

Chain-ladder is performed using quotients between accumulated amounts as link-ratios. That is, putting

$$
f(j)=\sum_{k=0}^{n-j} C(k, j) / \sum_{k=0}^{n-j} C(k, j-1) \quad \text { for } \quad j=1 \ldots n
$$

we have

$$
C(i, n)=C(i, n-i) f(n-i+1) f(n-i+2) \ldots f(n)
$$

The factors $f(j)$ describe the estimated distribution of the claims amounts over the development years, assumed to be one and the same for all accident years in the underlying model. The distribution of the accumulated amounts is given by $U(0), U(1), \ldots, U(n)$ where

$$
\begin{gathered}
U(j)=1 / f(j+1) f(j+2) \ldots f(n) \quad \text { for } \quad j=0 \ldots(n-1) \\
U(n)=1
\end{gathered}
$$

From (2) and (3) it follows that

$$
C(i, n-i)=C(i, n) U(n-i)
$$

Denote by $C(i)$ the sum of the first $(i+1)$ projected amounts. Also, denote by $C(\cdot, j)$ the $j$ th column sum (in the original triangle) and by $C^{\prime}(\cdot, j)$ the same sum with the term $C(n-j, j)$ omitted. That is

$$
C(\cdot, j)=\sum_{k=0}^{n-j} C(k, j) \quad C^{\prime}(\cdot, j)=\sum_{k=0}^{n-j-1} C(k, j)
$$




$$
C(i)=\sum_{k=0}^{i} C(k, n)
$$

By induction it is proved that

$$
C(i, n)=C(i-1) C(i, n-i) / C^{\prime}(\cdot, n-i)
$$

Formula (7) yields a rapid recursive calculation of the projections $C(i, n)$ for $i=1 \ldots n$. On the author's part it goes back to an observation made by Kjell Andersson (ANDERSSON, 1992).

From (4) and (7) we find

$$
\begin{gathered}
C^{\prime}(\cdot, n-i)=C(i-1) U(n-i) \\
C(\cdot, n-i)=C(i) U(n-i)
\end{gathered}
$$

Formulas (4) and (9) are contained in a theorem by Thomas Mack (MACK, 1991).

\section{NECESSARY AND SUFFICIENT CONDITIONS FOR EQUALITY}

We now bring all three triangles $C, D$ and $E$ into play. For $D$ and $E$ we use a notation corresponding to (5) and (6) above. The estimated cumulative distribution of claims amounts over development years, corresponding to $U$ for the triangle $C$, is denoted by $V$ for the triangle $D$.

Theorem 1: The necessary and sufficient conditions for the chain-ladder projections to be additive,

$$
E(i, n)=C(i, n)+D(i, n) \quad \text { for all } \quad i,
$$

is that for each positive $i$ at least one of the following two equalities (a) and (b) holds true

(a) $U(n-i)=V(n-i)$

(b) $C(i, n) /(C(0, n)+\ldots+C(i-1, n))=D(i, n) /(D(0, n)+\ldots+D(i-1, n))$

\section{Proof :}

We want to compare $E(i, n)$ with $C(i, n)+D(i, n)$.

For $i=0$, equality trivially holds as all three entities are then elements of the base triangles.

Now consider the case when $i$ is positive. Applying (7) to $E(i, n)$ and observing that the $E$-triangle is the sum of the $C$ - and $D$-triangles, we get

$$
E(i, n)=E(i-1)(C(i, n-i)+D(i, n-i)) /\left(C^{\prime}(\cdot, n-i)+D^{\prime}(\cdot, n-i)\right)
$$


We then apply (4) to the numerator and (8) to the denominator to get $E(i, n)=$ $E(i-1)(C(i, n) U(n-i)+D(i, n) V(n-i)) /(C(i-1) U(n-i)+D(i-1) V(n-i))$

Dividing through by $C(i, n)+D(i, n)$, and in the right hand member also both multiplying and dividing by $C(i-1)+D(i-1)$, we finally get

$$
E(i, n) /(C(i, n)+D(i, n))=Q(i) \times E(i-1) /(C(i-1)+D(i-1))
$$

where $Q(i)$ is the quotient between

$$
(C(i, n) U(n-i)+D(i, n) V(n-i)) /(C(i, n)+D(i, n))
$$

and

$$
(C(i-1) U(n-i)+D(i-1) V(n-i)) /(C(i-1)+D(i-1))
$$

The last two expressions are the averages of $U(n-i)$ and $V(n-i)$ using as weights, in the first case $C(i, n)$ and $D(i, n)$, and in the second case $C(i-1)$ and $D(i-1)$. Also remember that

$$
\begin{aligned}
& C(i-1)=C(0, n)+\ldots+C(i-1, n) \\
& D(i-1)=D(0, n)+\ldots+D(i-1, n) \\
& E(i-1)=E(0, n)+\ldots+E(i-1, n)
\end{aligned}
$$

Now the argument begins. First assume that the projections are additive so that

$$
E(i, n)=C(i, n)+D(i, n) \quad \text { for all } \quad i
$$

Then, from (10), $Q(i)=1$ for each positive $i$. According to (11) and (12) this means that either

$$
U(n-i)=V(n-i)
$$

or else, according to the interpretation of (11) and (12) as averages,

$$
C(i, n) / D(i, n)=C(i-1) / D(i-1)
$$

Conversely, if for each positive $i$ at least one of (17) and (18) is true, then $Q(i)=1$ and (16) follows by induction from (10) and the fact that (16) is true for $i=0$.

Condition (18) may be written

(19) $C(i, n) /(C(0, n)+\ldots+C(i-1, n))=D(i, n) /(D(0, n)+\ldots+D(i-1, n))$

This finishes the proof.

$C(i, n)$ and $D(i, n)$ are our estimated total claims amounts for accident year $i$ for the two subportfolios. We will use either member of (19) as a measure of the rate of increase (in claims volume) of the corresponding portfolio at accident year $i$.

If (17) holds for all $i$, or if (19) holds for all $i$, then the sufficient condition of Theorem 1 is fulfilled. We thus have the following two corollaries. 
Corollary 1: If the two subportfolios are equally long-tailed, then the chainladder projections are additive.

Corollary 2: If the two subportfolios have the same rate of increase for each accident year, then the chain-ladder projections are additive.

\section{SUFFICIENT CONDITIONS FOR INEQUALITY}

If, instead of (17), we have

$$
U(n-i) \leq V(n-i) \quad \text { for all positive } \quad i
$$

then the subportfolio $C$ will have an estimated accumulated distribution of claims amounts over development years which increases to one at a slower rate than that of $D$. We will then say that subportfolio $C$ is at least as long-tailed as subportfolio $D$.

If, instead of (19), we have

$$
C(i, n) / C(i-1) \leq D(i, n) / D(i-1) \quad \text { for all positive } \quad i
$$

we will say that $D$ increases at least as fast as $C$.

If this is the case, we will also have

$$
C(i, n) / D(i, n) \leq C(i-1) / D(i-1)
$$

Theorem 2: If one of two subportfolios is at least as long-tailed as, and increases (in claims volume) at least as fast as, the other one, then the chain-ladder projections of the combined portfolio are less than or equal to the sums of the corresponding projections of the two subportfolios. If one of the subportfolios is at least as long-tailed as the other one, while the latter increases at least as fast as the first one, then the chain-ladder projections of the combined portfolio are greater than or equal to the sums of the corresponding projections of the two subportfolios.

\section{Proof :}

If both (20) and (21) are fulfilled, then for the averages (11) and (12), which define the quotient $Q(i)$, we find

1) $U(n-i)$ is less than or equal to $V(n-i)$

2) The weight given to $U(n-i)$ in the numerator is less than or equal to the weight given to it in the denominator.

Thus $Q(i)$ is greater than or equal to one for all positive $i$. From (10) it then follows by induction that

$$
E(i, n) /(C(i, n)+D(i, n)) \geq 1 \quad \text { for all } \quad i
$$


Arguing in the same way, we see that if $C$ is at the same time more (or equally) long-tailed and faster (or equally) increasing as compared to $D$, that is (21) with reversed inequality sign and (20) hold true, then

$$
E(i, n) /(C(i, n)+D(i, n)) \leq 1 \quad \text { for all } \quad i
$$

This finishes the proof.

It may be noted that we have introduced only partial orderings between development triangles, in that the inequality signs in (20) and (21) in general may go in opposite directions for different $i$.

\section{CONCLUSIONS}

We have given a partial answer to the question of Section 1 on which method to use. The answer is almost self-evident, at least a posteriori. Assume, for instance, that we add together a long-tailed business which decreases in volume and a short-tailed, increasing one. The long-tailed character of the early accident years will give high lag-factors for the later development years. These lag-fators will then grossly overestimate the final amounts for the dominating short-tailed business of the later accident years. That is, the combined method will give the highest projections.

An example in the opposite direction may be a motor comprehensive account where no division is made between third party claims and hull damage claims. If the third party claims take an increasing share of the total business, a separation of the two types of claims into different development triangles would certainly have been desirable from a prudent point of view.

Even if a certain degree of prudence is to be recommended, the goal is not to have as large reserves as possible, but to have as correct reserves as possible. So, in conclusion, the lesson to be learnt from this exercise in the following one.

If one part of a portfolio can be assumed to differ significantly from the rest of the portfolio with respect to both long-tailedness and rate of change of the claims volume, that part should be treated separately in making chain-ladder projections. Returning to prudence, this is especially important if it is at the same time more long-tailed and faster increasing than the rest of the portfolio.

\section{REFERENCES}

AJNE, B. (1991) A Note on the Additivity of Chain-ladder Projections. Paper presented to the 23rd ASTIN Colloquium (Speaker's corner).

Andersson, K. (1992) A Direttissima in Chain-ladder and the Danger of Direttissimas. Personal communication.

Buhlmann, H. (1993) Claims Reserves: Theory and Practice. Mimeographed paper.

MACK, T. (1991) A Simple Parametric Model for Rating Automobile Insurance or Estimating IBNR Claims Reserves. ASTIN Bulletin 21, 93-109. 
APPENDIX

Below four pairs of simple development triangles $(C, D)$ are exhibited. For the first three pairs, chain-ladder projectins do add. This means that the projected amounts corresponding to the combined triangle $E$ are the sums of the corresponding projections for $C$ and $D$, in accordance with the results of Section 3 .

For the fourth pair, treating $C$ and $D$ separately will give more prudent projections for the combined portfolio. This means that the projected amounts of $E$ are less than or equal to the sums of the corresponding projections for $C$ and $D$ (with inequality sign in at least one place). This is in accordance with one of the two sufficient conditions of Section 4.

In all the cases there are three accident years 0,1 and 2. These are observed through development years 0 to 2,0 to 1 and 0 only, respectively. Thus, in the notation of the main paper, $n=2$. Projected amounts are shown within parentheses. The amounts in the third column are the chain-ladder projections.

\section{Case 1}

$C$

$\begin{array}{llllllll}100 & 200 & 300 & 100 & 250 & 375 & 200 & 450 \\ 100 & 300 & (450) & 100 & 250 & (375) & 200 & 550 \\ 160 & (400) & (600) & 100 & (250) & (375) & 260 & (650)\end{array}$

$\operatorname{Proj}(E)=\operatorname{Proj}(C)+\operatorname{Proj}(D)$

\section{D}

(375)
675

In this case $C$ and $D$ are equally long-tailed, the link-ratios (lag-factors) of formula (1) being $f(1)=2.5$ and $f(2)=1.5$. So projections add because of Corollary 1 of Section 3 .

\section{Case 2}

C

$\begin{array}{lll}100 & 200 & 300 \\ 100 & 300 & (450) \\ 260 & (650) & (975)\end{array}$

$D$

$\begin{array}{lllll}10 & 100 & 150 & 110 & 300 \\ 40 & 150 & (225) & 140 & 450 \\ 65 & (325) & (487.5) & 325 & (975)\end{array}$

450

$\operatorname{Proj}(E)=\operatorname{Proj}(C)+\operatorname{Proj}(D)$

In this case $C$ and $D$ have the same rate of increase (but not the same link ratios), as shown by the fact that the third columns are proportional to each other. So projections add because of Corollary 2 of Section 3 . 


\section{Case 3}

\begin{tabular}{lllllllll}
$C$ & & \multicolumn{3}{c}{$D$} & \multicolumn{5}{c}{$E$} \\
100 & 200 & 300 & 200 & 300 & 450 & 300 & 500 & 750 \\
200 & 400 & $(600)$ & 200 & 300 & $(450)$ & 400 & 700 & $(1050)$ \\
300 & $(600)$ & $(900)$ & 400 & $(600)$ & $(900)$ & 700 & $(1200)$ & $(1800)$
\end{tabular}

$\operatorname{Proj}(E)=\operatorname{Proj}(C)+\operatorname{Proj}(D)$

In this case none of the above-mentioned circumstances are present but additivity follows from Theorem 1 in Section 3. For $i=1$, the equality (17) is fulfilled, as the link ratio $f(2)=1.5$ for both $C$ and $D$, making $U(1)=V(1)$. For $i=2$, the equality (19) is fulfilled, as the quotient between the third element in column three and the sum of the first two ones is 1 for both $C$ and $D$.

$C$ is more long-tailed than $D$, as (20) is fulfilled with strict inequality for $i=2$. It is also faster increasing than $D$ as (21) is fulfilled with reversed inequality signs and strict inequality for $i=1$. This illustrates why strict inequalities cannot be introduced in Theorem 2 in Section 4, without adding the rather pointless requirement that the necessary and sufficient condition of Theorem 1 must be fulfilled.

\section{Case 4}

C

$\begin{array}{lll}100 & 250 & 375\end{array}$

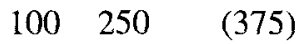

$100 \quad(250) \quad(375)$
D

$10 \quad 100$

$40 \quad 150$

$65 \quad(325)$
E

150

(225)

(487.5)

$\begin{array}{ll}110 & 350 \\ 140 & 400 \\ 165 & (495)\end{array}$

525

(742.5)

$\operatorname{Proj}(E)$ less than Proj $(C)+\operatorname{Proj}(D)$

This case illustrates a normal use of Theorem 2 in Section $4 . D$ is more long-tailed and faster increasing than $C$, and there is no equality sign in (21).

BJÖRN AJNE

Skandia, S-103 50 Stockhom. 